\title{
Respuesta Carta editor trabajo "Observaciones a la evaluación de propiedades psicométricas de validez y confiablidad de la Dental Anxiety Scale en adultos chilenos".
}

\author{
Response Letter to the editor work " Observations on the \\ evaluation of psychometric properties of validity and reliability of \\ the Dental Anxiety Scale in Chilean adults"
}

\author{
Matías Ríos-Erazo', Braulio Santibañez \\ de Investigación en Ciencias Odontológicas, \\ Facultad de Odontología, Universidad de Chile, \\ Chile. \\ 2. Internado Asistencial, Escuela de Pregrado, \\ Facultad de Odontología, Universidad de Chile, \\ Chile. \\ ${ }^{*}$ Correspondencia a: Andrea Herrera Ronda | \\ Dirección: Olivos 943, Independencia, Santiago, \\ Chile. | Teléfono: +562 29781844 | E-mail: \\ aherrera@odontologia.uchile.cl \\ Trabajo recibido 13/04/2021 \\ Aprobado para su publicación 13/04/2021
}

\author{
$z^{2}$, Paula van Treek ${ }^{1}$, Andrea Herrera-Ronda ${ }^{1 *}$, Gonzalo Rojas-Alcayaga1.
}

\section{Señor editor:}

Agradecemos la oportunidad de poder dar respuesta a los comentarios entregados por Romo-Pérez y De la Hoz (2020) los que valoramos enormemente ya que permiten la revisión de las propias prácticas, incorporar una mirada crítica y así generar conocimiento y hacer academía. Por ello, deseamos unirnos a esta experiencia de continuo aprendizaje y responder una a una las observaciones realizadas.

Establecer el tamaño de una muestra es un problema complejo, siendo objeto de estudio para muchos investigadores estadísticos ${ }^{(1)}$. En el trabajo realizado, donde se obtuvo un $n=$ 179 , se siguió la recomendación del trabajo clásico de Gorsuch ${ }^{(2)}$, que sugiere la proporción de 5 sujetos por ítem y un tamaño de muestra no menor a 100 personas, no obstante, es pertinente seguir las recomendaciones de mínimo 200 sujetos para un próximo estudio.

Resulta bastante evidente que las muestras más grandes son mejores que muestras pequeñas, más aún al momento de tratarse de estudios de validación, pero no se puede dejar de mencionar que muestras demasiado grandes también interfieren en la validez de datos de estudios de validación ${ }^{(3)}$. Sin embargo, es importante considerar el fenómeno a estudiar, puesto que no siempre es posible contar con una muestra representativa, que considere los sesgos y limitaciones del estudio de la ansiedad dental. Por esta misma razón, es que el tamaño muestral siempre fue considerado una limitación del estudio y como tal, fue señalado en la discusión(4).

El fenómeno de la ansiedad dental es aún poco estudiado en latinoamérica, en comparación con países desarrollados como Nueva Zelanda ${ }^{(5)}$ y Suecia(6), por lo que los resultados de sus investigaciones no son extrapolables a la realidad de nuestros países, tanto por factores culturales como por el sistema de salud. Es ésta una de las razones por la que es importante continuar generando conocimientos en esta área, sobretodo en nuestro contexto latinoamericano.

Respecto a los criterios para determinar el tamaño muestral, si bien estos no fueron mencionados en el artículo, sí están publicados en un estudio previo(7). Es importante mencionar que nuestro estudio es un análisis secundario de datos, por lo que el tamaño de la muestra fue calculado en base al outcome de ese estudio, a partir de un error de significancia de 0.05 , un poder estadístico de 0.95 , y un tamaño de efecto mediano, obteniendo un $\mathrm{n}$ de $130^{(7)}$.

Romo-Pérez y De la Hoz, basado en la literatura a la que hacen referencia( ${ }^{(8)}$, mencionan que la metodología Delphi no correspondería a un método adecuado para medir la validez de contenido, y que en realidad estaríamos midiendo la validez aparente. Carvajal y cols. (9) explicitan que la validez aparente es una forma de validez de contenido, y mencionan al método Delphi como un método legítimo para medir dicha validez, al igual que el modelo de estimación de magnitud, el modelo Fehring y la metodología $\mathrm{Q}^{(9)}$. Debido a que la referencia con la que estábamos trabajando es más antigua, consideramos adecuado incorporar las recomendaciones en los trabajos futuros en los que evaluemos tanto validez aparente como de constructo.

Respecto a los resultados reportados por el Análisis Factorial Confirmatorio, como bien mencionan Romo-Pérez y De la Hoz hacen falta algunos datos que mencionamos a continuación. Para la normalidad de la muestra se realizó la prueba de Kolmogorov-Smirnov obteniendo un Z: 1.269; $p=0.08$ asumiendo la normalidad del grupo evaluado, lo que además justifica el uso de Máxima verosimilitud (ML). El hecho de que la escala sea unidimensional y la variable de 5 categorías justifican el uso de la matriz de correlación de Pearson y el procedimiento $\mathrm{ML}^{(3)}$.

En la matriz de correlaciones, se obtuvo un determinante de la matriz de 0.237. Importante considerar que un determinante próximo a cero indica que las variables utilizadas están linealmente relacionadas, siendo pertinente la utilización del análisis factorial, siendo coherente con los valores obtenidos por el test de esfericidad de bartlett.

Respecto al índice de bondad de ajuste, se usó un índice inferencial absoluto, x2, y no comparativo ya que nos interesaba hacer inferencias respecto de la población y establecer 
en qué grado el modelo propuesto reproduce los datos, no comparar el modelo con otro alternativo(3).

Finalmente, agradecemos el aporte respecto al uso de Omega de McDonald en conjunto con el Alfa de Cronbach para determinar la confiabilidad de un instrumento, sin duda, lo incorporaremos a futuro para dar más solidez a nuestros resultados.

\section{Bibliografía}

1. Anthoine E, Moret L, Regnault A, Sbille V, Hardouin JB. Sample size used to validate a scale: a review of publications on newly-developed patient reported outcomes measures. Health Qual Life Outcomes. 2014;12(1):176.

2. Gorsuch R L. Factor Analysis. 2nd ed. Hillsdale, New Jersey/Lawrence, Erlbaum Associates;1983.

3. Abad F, Olea J, Ponsoda V, García C. Mediciones en ciencias sociales y de la salud. Ed. Síntesis, Madrid; 2011.

4. Ríos-Erazo M, Santibáñez B, van Treek P, Herrera-Ronda A, Rojas-Alcayaga

G. Validez de contenido, de constructo y confiabilidad del Dental Anxiety Scale en adultos chilenos. Int J Interdiscip Dent. 2020;13(1):9-12.

5. Thomson WM, Broadbent JM, Locker D, \& Poulton R. Trajectories of dental anxiety in a birth cohort. Community Dent Oral Epidemiol. 2009;37:209-19.
6. Nydell Helkimo A, Rolander B \& Koch G. Attitudes to dental visits in a child population attending public dental health care in Jönköping, Sweden-trends over 40 years. Eur Arch Paediatr Dent. 2021;22(3):351-360.

7. Rojas-Alcayaga GA, Alfaro K, Ríos-Erazo M, Herrera AC, Barahona P. Music distraction effectiveness in dental anxiety and treatment adherence in 6-year-old children: a randomized clinical trial. Int J Odontostomat. 2018;12(1):35-42.

8. Luján-Tangarife JA, Cardona-Arias JA. Construcción y validación de escalas de medición en salud: revisión de propiedades psicométricas. Arch Med. 2015;11(3):1. DOI: $10.3823 / 1251$

9. Carvajal A, Centeno C, Watson R, Martínez M, Sanz Rubiales Á. ¿Cómo validar un instrumento de medida de la salud?. Anales Sis San Navarra. 2011;34(1): 63-72. 